\title{
A Variational Approach to Boundary Value Problems for a Class of Nonlinear Systems of Mixed Type
}

\author{
H.-P. Gittel
}

\author{
Dedicated to Prof. Dr. Herbert Beckert \\ on the occasion of his 75 th birthday.
}

\begin{abstract}
A bstract. Transonic small disturbance theory in two dimensions leads to a nonlinear first-order system of mixed type. For such systems corresponding variational problems are formulated. These minimum problems are studied on closed sets of admissible functions satisfying certain boundedness constraints and entropy conditions. Existence theorems can be proved though the functionals need not be convex and the given sets are not compact. Furthermore, some properties of minimizers are derived.
\end{abstract}

Keywords: Transonic flows, generalized solutions, variational problems, entropy conditions, weak lower semi-continuity

AMS subject classification: Primary 76 H 05, secondary 49 J 99, 76 M 30

\section{Introduction}

A standard model in transonic gas dynamics is the irrotational steady flow of an inviscid gas past a profile in two dimensions. This flow can be described by the equations

$$
\begin{aligned}
& \frac{\partial}{\partial x_{1}}\left(\rho w_{1}\right)+\frac{\partial}{\partial x_{2}}\left(\rho w_{2}\right)=\operatorname{div}\left(\rho\left(|\mathbf{w}|^{2}\right) \mathbf{w}\right)=0 \quad \text { (continuity equation) } \\
& \frac{\partial}{\partial x_{1}} w_{2}-\frac{\partial}{\partial x_{2}} w_{1}=\operatorname{rot} \mathbf{w}=0 \quad \text { (conservation of vorticity) }
\end{aligned}
$$

where $\mathbf{w}=\left(w_{1}, w_{2}\right)$ is the velocity field and $\rho$ denotes the density related to $\mathbf{w}$ by the Bernoulli's law (cf., e.g., [8: Chapters I, IX and XII] for physical background). We assume that the flow is symmetric with respect to the $x_{1}$-axis, and uniformly parallel at infinity with the velocity $\left(w_{\infty}, 0\right)$ and the Mach number $M_{\infty}$ (cf. Figure 1).

H.-P. Gittel: Univ. Leipzig, Math. Institut, Augustusplatz 10/11, D - 04109 Leipzig 

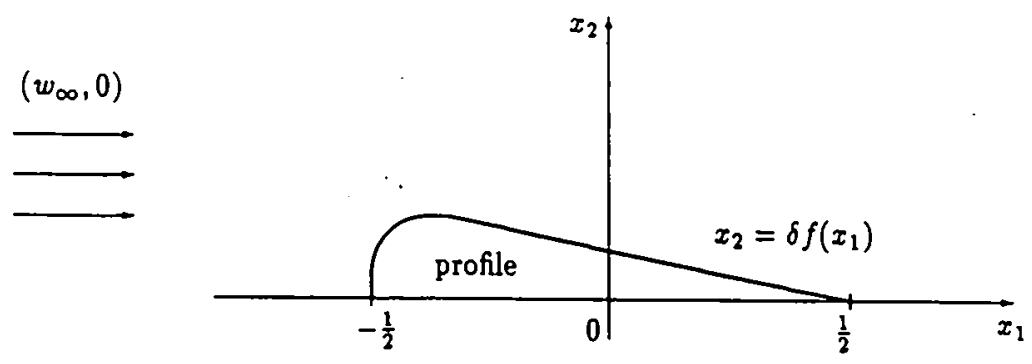

Figure 1

The equations (1.1) are studied in the upper half-plane $x_{2} \geq 0$. As usual (cf., e.g., $[14,15])$ the upper part of the airfoil is defined by a (sufficiently smooth) shape function $f$ and a thickness parameter $\delta>0$ :

$$
x_{2}=\delta f\left(x_{1}\right) \quad \text { for } \quad x_{1} \in\left[-\frac{1}{2},+\frac{1}{2}\right]
$$

with

$$
f\left(-\frac{1}{2}\right)=f\left(+\frac{1}{2}\right)=0, \quad f \geq 0, \quad \sup \left\{f\left(x_{1}\right) \mid x_{1} \in\left[-\frac{1}{2},+\frac{1}{2}\right]\right\}=1 .
$$

Let us consider the interval $\left(\delta, M_{\infty}\right)$ close to $(0,1)$. According to the well known tran. sonic expansion procedure (cf. [1: Chapter 3]) from (1.1) the two-dimensional small disturbance equations read as follows:

$$
\begin{aligned}
\frac{\partial}{\partial x_{1}}\left(K \tilde{w}_{1}-\frac{\gamma+1}{2} \tilde{w}_{1}^{2}\right)+\frac{\partial}{\partial \tilde{x}_{2}} \tilde{w}_{2} & =0 \\
\frac{\partial}{\partial x_{1}} \tilde{w}_{2}-\frac{\partial}{\partial \tilde{x}_{2}} \tilde{w}_{1} & =0 .
\end{aligned}
$$

follow. Here $K=\delta^{-2 / 3}\left(1-M_{\infty}^{2}\right)$ is the transonic similarity parameter, $\gamma>1$ the adiabatic constant and $\tilde{x}_{2}=\delta^{1 / 3} x_{2}$ is a new coordinate. The unknowns $\tilde{w}_{1}$ and $\tilde{w}_{2}$ are related to $w_{1}$ and $w_{2}$ by the asymptotic expansion in fractional powers of $\delta$ :

$$
w_{1}=w_{\infty}\left(1+\delta^{2 / 3} \tilde{w}_{1}\right)+\cdots \quad \text { and } \quad w_{2}=w_{\infty} \delta \tilde{w}_{2}+\cdots
$$

These new unknowns satisfy the boundary conditions

$$
\begin{aligned}
& \left(\tilde{w}_{1}, \tilde{w}_{2}\right) \longrightarrow(0,0) \text { as } x_{1}^{2}+\tilde{x}_{2}^{2} \rightarrow \infty \quad \text { (uniform flow at infinity) } \\
& \tilde{w}_{2}\left(x_{1}, 0\right)=f^{\prime}\left(x_{1}\right) \quad \text { for } \quad x_{1} \in\left[-\frac{1}{2},+\frac{1}{2}\right] \quad \text { (tangent flow). }
\end{aligned}
$$


Remark 1.1. Introducing the small disturbance potential $\Phi$ due to $\nabla \Phi=\widetilde{w}$ the first relation of (1.2) gives the Karman-Guderley equation (cf. [1: p. 51] and [13, 14]). The following investigations do work in an analogous way for this equation (and related ones), too.

In the sequel the parameters $w_{\infty}, \delta, \gamma$ and $K$ are assumed to be fixed. For simplicity of the mathematical discussion we carry out a further transformation of dependent and independent variables:

$$
\begin{aligned}
& x=x_{1} \\
& y=(\gamma+1)^{1 / 2} \tilde{x}_{2} \\
& u=K(\gamma+1)^{-1}-\tilde{w}_{1} \\
& v=-(\gamma+1)^{-1 / 2} \widetilde{w}_{2}
\end{aligned}
$$

and from (1.2) the following basic transonic system for $u=u(x, y)$ and $v=v(x, y)$ follows:

$$
\begin{array}{r}
u u_{x}+v_{y}=\operatorname{div}\left(\frac{1}{2} u^{2}, v\right)=0 \\
v_{x}-u_{y}=\operatorname{rot}(u, v)=0 .
\end{array}
$$

This system changes the type. Namely, it is

$\begin{array}{llll}\text { elliptic } & \text { iff } & u>0 & \text { (subsonic region) } \\ \text { parabolic } & \text { iff } & u=0 & \text { (sonic } \text { region) } \\ \text { hyperbolic } & \text { iff } & u<0 & \text { (supersonic } \text { region). }\end{array}$

To consider a boundary value problem for system (1.5) in a finite domain of the $(x, y)$ plane we choose a rectangle $\Omega=[-a, a] \times[0, b]$ with sufficiently large $a>0$ and $b>0$. Appropriate boundary conditons for $(u, v)$ due to (1.3) on $\partial \Omega=\Gamma_{1} \cup \Gamma_{2} \quad\left(\Gamma_{1}-\right.$ the vertical part and $\Gamma_{2}$ - the horizontal one) are

$$
\begin{aligned}
& (u, v) \cdot \mathrm{t}=0 \quad \text { on } \Gamma_{1} \\
& \left(\frac{1}{2} u^{2}, v\right) \cdot \mathbf{n}=g \quad \text { on } \quad \Gamma_{2}
\end{aligned}
$$

with

$$
\begin{aligned}
& g= \begin{cases}(\gamma+1)^{-1 / 2} f^{\prime}(x) & \text { for } x \in\left[-\frac{1}{2},+\frac{1}{2}\right], y=0 \\
0 & \text { otherwise }\end{cases} \\
& \mathbf{t}-\text { the unit tangent on } \partial \Omega \\
& \mathbf{n}-\text { the unit normal on } \partial \Omega
\end{aligned}
$$

(cf. Figure 2). 


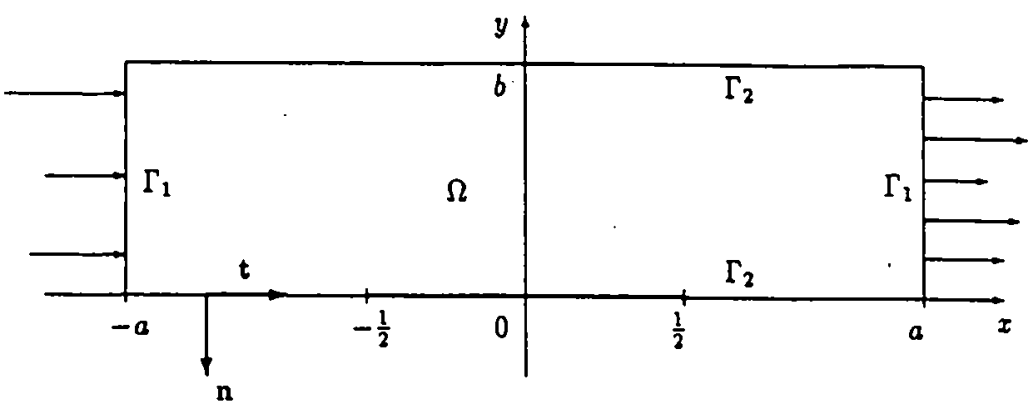

Figure 2

In this paper we study a more general boundary value problem then (1.5), (1.7):

Let $\Omega$ be a bounded domain in the $(x, y)$-plane with piecewise smooth boundary $\partial \Omega=\Gamma_{1} \cup \Gamma_{2} \cup \Gamma_{0}$, where $\Gamma_{1}$ and $\Gamma_{2}$ are open subsets of $\partial \Omega$, and $\mu_{1}\left(\Gamma_{0}\right)=0, \mu_{N}-$ the $N$-dimensional Lebesgue measure. We consider the first order system

$$
\begin{array}{r}
p(u)_{x}+v_{y}=0 \\
v_{x}-u_{y}=0
\end{array}
$$

in $\Omega$ with the following boundary conditions:

$$
\begin{array}{rll}
(u, v) \cdot \mathbf{t}=0 & \text { on } \quad \Gamma_{1} \\
(p(u), v) \cdot \mathbf{n}=g & \text { on } \quad \Gamma_{2} .
\end{array}
$$

We assume that $g$ and $p$ are given (sufficiently smooth) functions on $\Gamma_{2}$ and $\mathbb{R}$, respectively, and

$$
\begin{array}{rrr}
p^{\prime}(u) \geq c_{1} u & \text { for } \quad u>0 \\
0>p^{\prime}(u) \geq c_{2} u & \text { for } \quad u<0
\end{array}
$$

with positive constants $c_{1}$ and $c_{2}$. The inequalities (1.10) imply the change of type of system (1.8) as established in (1.6). Moreover, we suppose the growth condition

$$
|p(u)| \leq c_{3}\left(1+|u|^{2}\right) \quad \text { for all } \quad u \in \mathbb{R}
$$

with some positive constant $c_{3}$.

Remark 1.2. Our system (1.8) corresponds to the well known p-system (cf., e.g., [19: p. 258]) extensively discussed for the hyperbolic case in [16: Chapter 17.A]. The basic transonic system (1.5) is a special case with $p(u)=\frac{1}{2} u^{2}$.

In transonic flows we have to take into account that there exist subsonic regions as well as supersonic ones in $\Omega$ and that shocks with jumps in $(u, v)$ occur. From the mathematical point of view these facts causes many difficulties in the existence proofs for system (1.8) and, of course, for the full system (1.1). These questions are still open 
also in the case of generalized solutions. Steps in this direction were done by Feistauer, Morawetz, Nečas, Pogu and the author (cf. [2 - 7, 9, 10, 12 - 15]).

Since discontinuous solutions of system (1.8) are allowed the theory of nonlinear hyperbolic systems (cf., e.g., [16]) says that solutions "without physical sense" (rarefraction shocks) can occur. To select the "physically right" ones the solutions of system (1.8) must additionally satisfy an entropy condition. In this paper we confine ourselves to the condition

$$
-u_{\mathbf{x}} \leq M(x, y)
$$

in the distributional sense with a given non-negative function $M \in L^{r}(\Omega)(r>1)$. In the original variables due to (1.4) this condition reads as

$$
\frac{\partial \tilde{w}_{1}}{\partial x_{1}} \leq M\left(x_{1},(\gamma+1)^{1 / 2} \tilde{x}_{2}\right)
$$

It is admissible (for $M \equiv$ const) in the sense of Warnecke (cf. [19: Lemma 5.3]) and implies that the flow velocity in the distinguished direction $x_{1}$ is decreasing through a shock (cf. [1: p. 55] and [19: Subsection 3.2]).

We deal with the boundary value problem (1.8), (1.9) in a weak form and with the corresponding variational problem. In Section 2 we study this minimum problem on a closed convex set which is given by $L^{\infty}$-bounds and the entropy condition (1.12). Here the functional considered need not be convex as it is in the subsonic case. But, using some compensated compactness arguments the weak lower semi-continuity of this functional can be proved. In Section 3 we minimize the same functional over a modified set where the constraints for admissible functions are weakened. Instead of the entropy condition (1.12) we use its local version in the sense of [7]. Furthermore, the established estimates for that functional enables us in Section 4 to discuss some properties of minimizers. We obtain results on uniqueness and on relations to the boundary value problem (1.8), (1.9).

Throughout the paper only the case of two dimensions is considered to make clear the underlying ideas. Generalizations to higher dimensions are evident (cf. Remark 4.6./ii)).

\section{Variational formulation}

To formulate a generalized problem to problem (1.8), (1.9) we introduce the space

$$
\mathcal{V}=\left\{\begin{array}{l|l}
(h, k) \in L^{3}(\Omega) \times L^{2}(\Omega) & \begin{array}{c}
\operatorname{rot}(h, k)=0 \text { (in distr. sense) } \\
(h, k) \cdot \mathbf{t}=0 \text { in } H^{-1 / 2}\left(\Gamma_{1}\right)
\end{array}
\end{array}\right\} .
$$

This is a closed linear subspace of $L^{3}(\Omega) \times L^{2}(\Omega) \subset L^{2}\left(\Omega, R^{2}\right)$, and $H^{-1 / 2}\left(\Gamma_{1}\right)$ is the dual space of

$$
H^{1 / 2}\left(\Gamma_{1}\right)=\left\{\psi \in H^{1 / 2}(\partial \Omega) \mid \operatorname{supp} \psi \subset \Gamma_{1}\right\} .
$$

Due to the inclusions $h, k, \operatorname{rot}(h, k) \in L^{2}(\Omega)$ the functional $\langle(h, k) \cdot \mathrm{t}, \psi\rangle_{\Gamma^{\prime}}$ can be defined for all $\psi \in H^{1 / 2}\left(\Gamma^{\prime}\right), \Gamma^{\prime}$ being an open subset of $\partial \Omega$, in the usual way (cf., e.g., [18: pp. 9 - 13]). 
Note that $\nabla \phi \in \mathcal{V}$ for a smooth function $\phi$ which vanishes in a neighbourhood of $\Gamma_{1}$. For such function $\phi$ and smooth functions $u$ and $v$ the second boundary condition in (1.9) is equivalent to

$$
\begin{aligned}
\int_{\Gamma_{2}}(p(u) \dot{y}(\tau)-v \dot{x}(\tau)) \phi d \tau & =\int_{\Gamma_{2}} g \phi d s \\
& =-\int_{\Gamma_{2}}\left(\int_{\alpha}^{\tau} g(x(\sigma), y(\sigma)) \sqrt{\dot{x}^{2}+\dot{y}^{2}}(\sigma) d \sigma\right) \nabla \phi \cdot \mathbf{t} d s \\
& =:\langle\nabla \phi \cdot \mathbf{t}, G\rangle_{\Gamma_{2}}
\end{aligned}
$$

with $(x, y)=(x(\tau), y(\tau)) \quad(\tau \in(\alpha, \beta))$ being a representation of $\Gamma_{2}$, and $G$ a function on $\Gamma_{2}$ choosen in natural way. The functional $\langle(h, k) \cdot \mathbf{t}, G)_{\mathrm{r}_{2}}$ is defined for $(h, k) \in \mathcal{V}$, and hence, there exists a unique element $\left(u_{0}, v_{0}\right) \in \mathcal{V}$ such that

$$
\langle(h, k) \cdot \mathbf{t}, G\rangle_{\Gamma_{2}}=\iint_{\Omega}(h, k) \cdot\left(u_{0}, v_{0}\right) d x d y
$$

So, we are led to the following definition.

Definition 2.1. An element $(u, v) \in \mathcal{V}$ is called generalized solution of the boundary value problem (1.8), (1.9) if

$$
\iint_{\Omega}\left((p(u), v)-\left(u_{0}, v_{0}\right)\right) \cdot(h, k) d x d y=0 \quad \text { for all }(h, k) \in \mathcal{V}
$$

It is casy to see that (2.4) is the Euler-Lagrange equation for the variational problem

$$
J(u, v):=\iint_{\Omega}\left(P(u)+\frac{1}{2} v^{2}-\left(u u_{0}+v v_{0}\right)\right) d x d y \longrightarrow \min _{(u, v) \in \mathcal{V}}
$$

with $P(u)=\int_{0}^{u} p(\sigma) d \sigma$ being the primitive of $p$. But we seek a solution $(u, v)$ of problem (2.5) which, in addition, is bounded and satisfies the entropy condition (1.12) in the form

$$
\iint_{\Omega} u \phi_{x} d x d y \leq \iint_{\Omega} M \phi d x d y
$$

for all

$$
\phi \in\left(C_{0}^{\infty}(\Omega)\right)_{+}=\left\{\phi \in C^{\infty}(\Omega) \mid \operatorname{supp} \phi \subset \subset \Omega \text { and } \phi \geq 0\right\}
$$

Therefore we study this minimum problem over the set

$$
\mathcal{K}=\mathcal{K}(m, M)=\left\{\begin{array}{l|l}
(h, k) \in \mathcal{V} \mid \begin{array}{r}
|h|,|k| \leq m \text { a.e. on } \Omega \\
(h, k) \text { satisfying }(2.6)
\end{array}
\end{array}\right\}
$$

This is a non-empty, closed, convex subset of $\mathcal{V}$ bounded in $L^{\infty}\left(\Omega, R^{2}\right)$. 
Remark 2.2. i) In the case of the basic transonic system (1.5) the a priori the $L^{\infty}$ bound on $(h, k)$ in (2.7) means an additional constraint for admissible small disturbances of the velocity field. Such a constraint may be caused by the range of validity of the physical model and of the transonic expansion procedure. From the mathematical point of view we are able to weaken this constraint (cf. Section 3 ).

ii) This variational approach is well known for the elliptic case, i.e. for pure subsonic flow problems. Defining

$$
\mathcal{K}_{\text {ell }}=\{(h, k) \in \mathcal{V}|| h|,| k \mid \leq m \text { and } h>0 \text { a.e. on } \Omega\}
$$

the minimum problem

$$
J(u, v) \longrightarrow \min _{(u, v) \in \mathcal{K}_{\mathrm{ell}}}
$$

posses a unique solution because the functional $J$ is strictly convex, continuous and bounded on $\mathcal{K}_{\text {ell }}$ (cf., e.g., [20: Theorems 38.A and 38.C]). Here no entropy condition is needed. To extend this approach to transonic flow problems this condition is necessary to compensate the failure of convexity of $J$.

To prove that the functional $J$ is weakly lower semi-continuous on $\mathcal{K}$ we estimate the difference of two values of $J$ from below. Throughout the paper we use $\|\cdot\|_{q}$ as abbreviation'for the $L^{q}(\Omega)$-Norm, $1 \leq q \leq \infty$.

Lemma 2.3. For arbitrary elements $(h, k),(u, v) \in \mathcal{V}$ we have

$$
\begin{aligned}
J(h, k)- & J(u, v) \\
\geq & D J(u, v ; h-u, k-v)+\frac{1}{2}\|k-v\|_{2}^{2} \\
& +\iint_{\Omega}\left(\frac{c_{1}}{6}(h+2 u)+\frac{\left(c_{2}-c_{1}\right)^{+}}{6}\left(h^{-}+2 u^{-}\right)\right)(h-u)^{2} d x d y
\end{aligned}
$$

with $\gamma^{+}=\max \{0, \gamma\}$ and $\gamma^{-}=\min \{0, \gamma\}$ for $\gamma \in \mathbb{R}$.

Proof. Due to the definition of the functional $J$ in (2.5) we have

$$
\begin{array}{r}
J(h, k)-J(u, v)=\iint_{\Omega}\left((P(h)-P(u))+\frac{1}{2}\left(k^{2}-v^{2}\right)\right. \\
\left.-(h-u) u_{0}-(k-v) v_{0}\right) d x d y .
\end{array}
$$

To get an estimate from below for the integrand of the right-hand side we study its first term

$$
P(h)-P(u)=p(u)(h-u)+\left(\int_{0}^{1}(1-\theta) p^{\prime}(u+\theta(h-u)) d \theta\right)(h-u)^{2} .
$$

Decomposing $p^{\prime}$ the assumption (1.10) gives

$$
\begin{aligned}
p^{\prime}(\gamma)=\left(p^{\prime}(\gamma)\right)^{+}+\left(p^{\prime}(\gamma)\right)^{-} & \geq c_{1} \gamma^{+}+c_{2} \gamma^{-} \\
& =c_{1} \gamma+\left(c_{2}-c_{1}\right) \gamma^{-} \\
& \geq c_{1} \gamma+\left(c_{2}-c_{1}\right)^{+} \gamma^{-}
\end{aligned}
$$


for $\gamma \in \mathbb{R}$. Setting $\gamma^{\circ}=u+\theta(h-u)=\theta h+(1-\theta) u$ and using the concavity of the function $\gamma \mapsto \gamma^{-}$we obtain

$$
\begin{aligned}
& \int_{0}^{1}(1-\theta) p^{\prime}(u+\theta(h-u)) d \theta \\
& \geq c_{1} \int_{0}^{1}(1-\theta)(\theta h+(1-\theta) u) d \theta \\
& \quad+\left(c_{2}-c_{1}\right)^{+} \int_{0}^{1}(1-\theta)\left(\theta h^{-}+(1-\theta) u^{-}\right) d \theta \\
& =\frac{c_{1}}{6}(h+2 u)+\frac{\left(c_{2}-c_{1}\right)^{+}}{6}\left(h^{-}+2 u^{-}\right) .
\end{aligned}
$$

Finally, from (2.9) and (2.10) inequality (2.8) follows if we rewrite $k^{2}-v^{2}=2 v(k-v)+$ $(k-v)^{2}$

Remark 2.4. Inequality (2.8) is a generalization of the corresponding one in the convex case of the functional $J$ (i.e. $h, u>0$ ). In this case the term with $c_{2}$ disappears.

With the help of simple methods from compensated compactness theory we obtain a compactness property of the set $\mathcal{K}$ defined by (2.7) which is crucial in the existence proof below.

Lemma 2.5. Let $\left\{\left(u_{n}, v_{n}\right)\right\} \subset \mathcal{K}$ be a sequence weakly converging in $L^{2}\left(\Omega, R^{2}\right)$. Then the sequence $\left\{u_{n}\right\}$ converges strongly in $L^{q}(\Omega)$ for each $q \in[1, \infty)$.

Proof. a) For $\phi \in C_{0}^{\infty}(\Omega)$ we define the functionals

$$
F_{n}(\phi)=\iint_{\Omega}\left(M \phi-u_{n} \phi_{x}\right) d x d y
$$

and get the estimate

$$
\left|F_{n}(\phi)\right| \leq\|M\|_{r}\|\phi\|_{r^{*}}+\left\|u_{n}\right\|_{3}\left\|\phi_{x}\right\|_{3 / 2} \leq C\left(\|M\|_{r}+m \mu_{2}(\Omega)^{1 / 3}\right)\|\phi\| W^{1, p}(\Omega)
$$

with $\frac{1}{r}+\frac{1}{r^{*}}=1$ and a constant $C=C(r, p)$. Here we have used the inequality

$$
\left\|u_{n}\right\|_{\infty} \leq m
$$

due to the definition (2.7) of the set $\mathcal{K}$ and the Sobolev imbedding (cf. [17: p. 213])

$$
W_{0}^{1, p}(\Omega) \hookrightarrow L^{r^{*}}(\Omega) \quad \text { for } p \in\left[p_{0}, 2\right] \text { with } p_{0}=\max \left\{\frac{2 r^{*}}{2+r^{*}}, \frac{3}{2}\right\}<2 \text {. }
$$

Hence, the sequence $\left\{F_{n}\right\}$ is bounded in $W^{-1, p^{*}}=\left(W_{0}^{1, p}(\Omega)\right)^{*}\left(\frac{1}{p}+\frac{1}{p^{*}}=1\right)$. Moreover, the entropy condition (2.6) gives $F_{n}(\phi) \geq 0$ for all $\phi \in\left(C_{0}^{\infty}(\Omega)\right)_{+}$, and Murat's result 
[11: p. 319] yields the compactness of the sequence $\left\{F_{n}\right\}$ in $W^{-1, q}$ for each $q<p^{*}$. If we choose $p<2$ or, equivalently, $p^{*}>2$ we obtain that $\left\{F_{n}\right\}$, and consequently, $\left\{\frac{\partial}{\partial x} u_{n}\right\}=\left\{\operatorname{div}\left(u_{n}, 0\right)\right\}$ is compact in $W^{-1,2}$.

b) Recalling the definition (2.1) of the space $\mathcal{V}$ we have $\operatorname{rot}\left(u_{n}, v_{n}\right)=0$ for all $n$. The Div-Curl lemma of Tartar (cf. [17: p. 28]) implies the weak convergence

$$
\left(u_{n}, 0\right) \cdot\left(u_{n}, v_{n}\right)=u_{n}^{2}-(u, 0) \cdot(u, v)=u^{2} \quad \text { (in the distributional sense) }
$$

if $u_{n} \rightarrow u$ and $v_{n} \rightarrow v$ in $L^{2}(\Omega)$, as $n \rightarrow \infty$. From this and (2.11) the assertion follows

Theorem 2.6. Let the functional $J$ and the set $\mathcal{K}$ be given by (2.5) and (2.7), respectively. Then:

a) The functional $J$ is weakly lower semi-continuous on $\mathcal{K}$.

b) The variational problem

$$
J(u, v) \longrightarrow \min _{(u, v) \in \mathcal{K}}
$$

has a solution.

c) Each minimizing sequence from $\mathcal{K}$ for $J$ possesses a subsequence converging to a solution of problem (2.12) in $\mathcal{V} \cap L^{q}\left(\Omega, R^{2}\right)$ for each $q \in[1, \infty)$.

Corollary 2.7. Any solution $(u, v) \in \mathcal{K}$ of problem (2.12) satisfies the corresponding variational inequality

$$
\begin{aligned}
D J(u, v ; h & -u, k-v) \\
& =\iint_{\Omega}\left(\left(p(u)-u_{0}\right)(h-u)+\left(v-v_{0}\right)(k-v)\right) d x d y \\
& \geq 0
\end{aligned}
$$

for all $(h, k),(u, v) \in \mathcal{K}$.

Proof a) Let $\left\{\left(u_{n}, v_{n}\right)\right\} \subset \mathcal{K}$ be à sequence weakly converging in $L^{2}\left(\Omega, \mathbb{R}^{2}\right)$, i.e. $u_{n} \rightarrow u$ and $v_{n} \rightarrow v$ in $L^{2}(\Omega)$ as $n \rightarrow \infty$. Since the set $\mathcal{K}$ is convex and closed in $\mathcal{V}$ the inclusion $(u, v) \in \mathcal{K}$ is true. Inequality $(2.8)$ with $(h, k)=\left(u_{n}, v_{n}\right)$ yields

$$
J\left(u_{n}, v_{n}\right)-J(u, v) \geq D J\left(u, v ; u_{n}-u, v_{n}-v\right)+\frac{1}{2}\left\|v_{n}-v\right\|_{2}^{2}-c\left\|u_{n}-u\right\|_{2}^{2}
$$

where the estimates $\left|u_{n}\right|,|u| \leq m$ a.e. on $\Omega$ are used and $c=\frac{m}{2}\left(c_{1}+\left(c_{2}-\dot{c}_{1}\right)^{+}\right)$. To pass to the limit $n \rightarrow \infty$ in (2.14) we only have to consider the last term of the right-hand side. This term is negative, but due to Lemma 2.5 it tends to 0 such that $\liminf _{n \rightarrow \infty} J\left(u_{n}, v_{n}\right)-J(u, v) \geq 0$

b) This assertion and Corollary 2.7 are standard results following from the properties of the functional $J$ and the set $\mathcal{K}$ (cf., e.g., [20: Proposition 38.12/(d)]). 
c) Let $\left\{\left(u_{n}, v_{n}\right)\right\} \subset \mathcal{K}$ and $J\left(u_{n}, v_{n}\right) \rightarrow \alpha=\min \mathcal{K} J$. Selecting a suitable subsequence we can assume $u_{n}-u$ and $v_{n} \rightarrow v$ in $L^{2}(\Omega)$. Furthermore, from Lemma 2.5 we get $u_{n} \rightarrow u$ in $L^{q}(\Omega)$, and hence,

$$
\alpha-J(u, v) \geq \frac{1}{2} \limsup _{n \rightarrow \infty}\left\|v_{n}-v\right\|_{2}^{2} \geq 0
$$

when we pass to the limit $n \rightarrow \infty$ in (2.14). Note that $J(u, v) \geq \alpha$ to derive $J(u, v)=\alpha$ and $v_{n} \rightarrow v$ in $L^{q}(\Omega)$ for $q=2$. For $q>2$ the assertion follows from the boundness of the sequence $\left\{v_{n}\right\}$ in $L^{\infty}(\Omega)$

The variational inequality (2.13) can be considered as a further generalization of the weak problem (2.4). It is immediately to see that a solution $(u, v) \in \mathcal{K}$ of inequality (2.13) which lies in the interior of $\mathcal{K}$ satisfies relation (2.4). But the needed estimates for $(u, v)$ to lay in the interior of $\mathcal{X}$ cannot be derived a priori. The question arises if it is possible to enlarge the class $\mathcal{K}$ of admissible functions for the variational problem (2.12). To this purpose we analyze carefully the proof of Theorem 2.6 to weaken the constraints established in the definition (2.7) of the class $\mathcal{K}$.

\section{Local entropy condition}

In this section we make use of the concept of local entropy conditions introduced in [7] but in a more natural way.

Definition 3.1. Let $(u, v) \in \mathcal{V}$. In accordance with the assumption (1.6) of the change of type of system (1.8) we define the subsets

$$
\begin{aligned}
\Omega^{+}(u, v) & =\{(x, y) \in \Omega \mid u>0\} \\
\Omega^{0}(u, v) & =\{(x, y) \in \Omega \mid u=0\} \\
\Omega^{-}(u, v) & =\{(x, y) \in \Omega \mid u<0\}
\end{aligned}
$$

denoting the subsonic, sonic, and supersonic region of $(u, v)$, respectively.

Definition 3.2. We say that the element $(u, v) \in \mathcal{V}$ satisfies the local entropy condition if

$$
\iint_{\Omega} u^{-} \phi_{x} d x d y=\iint_{\Omega^{-}} u \phi_{x} d x d y \leq \iint_{\Omega} M \phi d x d y
$$

for all $\phi \in\left(C_{0}^{\infty}(\Omega)\right)_{+}$, where $u^{-}=\min \{0, u\}, \Omega^{-}=\Omega^{-}(u, v)$ and $M \in L^{r}(\Omega)(r>1)$ is a given non-negative function.

Remark 3.3. Contrary to the global entropy condition (2.6) condition (3.2) means $-u_{x} \leq M$ in the distributional sense not on the whole of $\Omega$ but only on the supersonic (or hyperbolic) region $\Omega^{-}$.

Now, instead of (2.12) we study the variational problem

$$
J(u, v) \longrightarrow \min _{(u, v) \in \mathcal{K}_{l \diamond c}}
$$


with

$$
\mathcal{K}_{\text {loc }}=\mathcal{K}_{\text {loc }}(s, m, M)=\left\{(h, k) \in \mathcal{V} \mid\left\|h^{-}\right\|_{\mathrm{s}} \leq m,(h, k) \text { satisfying (3.2) }\right\}
$$

for given constants $s>3, m \geq 0$ and the function $M$ from (3.2). Here we have additionally weakened the $L^{\infty}$-constraints in the definition (2.7) of the class $\mathcal{K}$. Hence, all elements $(u, v) \in \mathcal{V}$ with $u \geq 0$ a.e. on $\Omega$ (i.e. for which system (1.8) is not of hyperbolic type) are a priori admissible for problem (3.3).

It is easy to see that $\mathcal{K}_{\text {loc }}$ is again a non-empty closed subset of $\mathcal{V}$. But, due to the non-linearity of the local entropy condition (3.2) the set $\mathcal{K}_{\text {loc }}$ is not convex. Applying the same compensated compactness arguments as for the set $\mathcal{K}$ we get the following compactness property.

Lemma 3.4. Let $\left\{\left(u_{n}, v_{n}\right)\right\} \subset \mathcal{K}_{\text {loc }}$ be a sequence weakly converging in $\mathcal{V}$ to $(u, v)$. Then we have

(i) the strong convergence $u_{n}^{-} \rightarrow u^{-}$in $L^{q}(\Omega)$ for each $q \in[1, s)$

(ii) the weak convergence $u_{n}^{+} \rightarrow u^{+}$in $L^{3}(\Omega)$

(iii) the strong convergence $u_{n} \rightarrow u$ in $L^{q}\left(\Omega^{0-}\right), \Omega^{0-}=\Omega^{0}(u, v) \cup \Omega^{-}(u, v)$ for each $q \in[1,3)$, as $n \rightarrow \infty$.

Proof. a) Substituting $u_{n}$ by $u_{n}^{-}$in part a) of the proof of Lemma 2.5 we get by the same arguments that $\left\{\frac{\partial}{\partial x} u_{n}^{-}\right\}=\left\{\operatorname{div}\left(u_{n}^{-}, 0\right)\right\}$ is compact in $W^{-1,2}$. Note that

$$
\left\|u_{n}^{-}\right\|_{s} \leq m \quad(s>3)
$$

due to the definition (3.4) of the set $\mathcal{K}_{\text {loc }}$.

b) Let $\left\{u_{n^{\prime}}^{-}\right\} \subseteq\left\{u_{n}^{-}\right\}$be a subsequence with $u_{n^{\prime}}^{-} \rightarrow a$ in $L^{s}(\Omega)$ as $n^{\prime} \rightarrow \infty$. Since $\operatorname{rot}\left(u_{n^{\prime}}, v_{n^{\prime}}\right)=0$ the Div-Curl lemma yields $\left(u_{n^{\prime}}^{-}, 0\right) \cdot\left(u_{n^{\prime}}, v_{n^{\prime}}\right) \rightarrow(a, 0) \cdot(u, v)$ or, equivalently,

$$
u_{n^{\prime}}^{-} u_{n^{\prime}}=\left(u_{n^{\prime}}^{-}\right)^{2} \rightarrow a u \quad \text { in the distributional sense. }
$$

c) To show that $a=u^{-}$a.e. on $\Omega$ we use the convexity of the functions $\gamma \mapsto \gamma^{2}$ and $\gamma \mapsto-\gamma^{-}$. The weak lower semi-continuity of the corresponding functionals implies the inequalities

$$
\begin{aligned}
a^{2} & \leq a u \\
-u^{-} & \leq-a
\end{aligned}
$$

a.e. on $\Omega$. Let $\Omega^{\prime} \subseteq \Omega$ such that $u^{-}>a$ on $\Omega^{\prime}$. Then $a<0$ and $a^{2}>a u^{-} \geq a u$ on $\Omega^{\prime}$ which contradicts (3.7). Hence, $\mu_{2}\left(\Omega^{\prime}\right)=0$ and together with (3.8) we obtain $a=u^{-}$ a.e. on $\Omega$.

d) From (3.6) and the result above it follows that $u_{n}^{-} \rightarrow u^{-}$and $\left(u_{n}^{-}\right)^{2} \rightarrow\left(u^{-}\right)^{2}$ as $n \rightarrow \infty$. Because of (3.5) interpolation gives statement (i). Furthermore, we get $u_{n}^{+}=u_{n}-u_{n}^{-} \rightarrow u-u^{-}=u^{+}$in $L^{3}(\Omega)$, i.e. statement (ii).

e) Recalling the definition of the set $\Omega^{0-}$ and (3.1) we have

$$
u_{n}^{-} \rightarrow u^{-}=u \quad \text { and } \quad\left|u_{n}^{+}\right|=u_{n}^{+} \rightarrow u^{+}=0 \quad \text { in } L^{3}\left(\Omega^{0-}\right),
$$

i.e. $u_{n}^{+} \rightarrow 0$ in $L^{1}\left(\Omega^{0-}\right)$. Interpolation and $u_{n}=u_{n}^{+}+u_{n}^{-}$yields statement (iii) 
As an immediate consequence of Lemma 3.4 we get that every bounded subset of $\mathcal{K}_{\text {loc }}$ is weakly compact in $\mathcal{V}$. That means that each bounded sequence from $\mathcal{K}_{\text {loc }}$ possesses a subsequence weakly converging in $\mathcal{V}$ with limit point in $\mathcal{K}_{\text {loc }}$. This result enables us to derive an existence theorem for the variational problem (3.3).

Theorem 3.5. The assertions a) - c) of Theorem 2.6 are valid if $\mathcal{K}$ is substituted by $\mathcal{K}_{\text {loc }}$ defined by (3.4), and $L^{q}\left(\Omega, \mathbb{R}^{2}\right)$ is substituted by $L^{q}(\Omega) \times L^{2}(\Omega)$ with $q \in[1, s)$.

Proof. a1) To prove the weak lower semi-continuity of the functional $J$ on $\mathcal{K}_{\text {loc }}$ we consider a sequence $\left\{\left(u_{n}, v_{n}\right)\right\} \subset \mathcal{K}_{\text {loc }}$ weakly converging in $\mathcal{V}$ to $(u, v)$. We have the inclusion $(u, v) \in \mathcal{K}_{\text {loc }}$. Inequality $(2.8)$ with $(h, k)=\left(u_{n}, v_{n}\right)$ gives

$$
\begin{aligned}
J\left(u_{n}, v_{n}\right)- & J(u, v) \\
\geq & D J\left(u, v ; u_{n}-u, v_{n}-v\right)+\frac{1}{2}\left\|v_{n}-v\right\|_{2}^{2} \\
& +\frac{c_{1}}{6} \iint_{\Omega}\left(u_{n}^{+}+2 u^{+}\right)\left(u_{n}-u\right)^{2} d x d y+I_{n}\left(\Omega^{+}\right)+I_{n}\left(\Omega^{0-}\right)
\end{aligned}
$$

where

$$
\begin{aligned}
I_{n}\left(\Omega^{\prime}\right) & =c \iint_{\Omega^{\prime}}\left(u_{n}^{-}+2 u^{-}\right)\left(u_{n}-u\right)^{2} d x d y \quad \text { for } \Omega^{\prime} \subseteq \Omega, \Omega^{+}=\Omega^{+}(u, v) \\
c & =\frac{1}{6}\left(c_{1}+\left(c_{2}-c_{1}\right)^{+}\right) .
\end{aligned}
$$

a2) To pass to the limit $n \rightarrow \infty$ in (3.9) only the last two terms of the right-hand side of (3.9) are of interest. Due to Lemma 3.4/(i) we have $u_{n}^{-}+2 u^{-} \rightarrow 3 u^{-}=0$ in $L^{3}\left(\Omega^{+}\right)$. Since $\left(u_{n}-u\right)^{2}$ is bounded in $L^{3 / 2}(\Omega)=\left(L^{3}(\Omega)\right)^{*}$ the integral $I_{n}\left(\Omega^{+}\right)$ converges to 0 . To get $\lim _{n \rightarrow \infty} I_{n}\left(\Omega^{0-}\right)=0$ we apply Lemma $3.4 /($ iii) and obtain $\left(u_{n}-u\right)^{2} \rightarrow 0$ in $L^{q}\left(\Omega^{0-}\right)$ for $q \in\left[\frac{1}{2}, \frac{3}{2}\right)$. Using the definition (3.4) we see that $u_{n}^{-}+2 u^{-}$ are bounded in $L^{s}(\Omega)$ with $s>3$, uniformly in $n$, and the desired convergence follows.

b1) In the existence proof for the solution of the variational problem (3.3) we have to take into account that $\mathcal{K}_{\text {loc }}$ is an unbounded set of $\mathcal{V}$. But, it is easy to get the coerciveness of the functional $J$ on $\mathcal{K}_{\text {loc }}$. Namely, (2.8) with $(h, k) \in \mathcal{K}_{\text {loc }}$ and $(u, v)=(0,0)$ implies

$$
\begin{aligned}
J(h, k)-J(0,0) \geq & \iint_{\Omega}\left(\left(p(0)-u_{0}\right) h-v_{0} k\right)(h-u)^{2} d x d y \\
& +\frac{1}{2}\|k\|_{2}^{2}+\frac{c_{1}}{6} \iint_{\Omega} h^{+} h^{2} d x d y+c \iint_{\Omega} h^{-} h^{2} d x d y
\end{aligned}
$$

where the constant $c$ is from (3.10). Using the definition of $J$ in (2.5), Young's inequality and the representation $h^{+}=|h|+h^{-}$we derive

$$
\begin{aligned}
J(h, k) \geq- & \frac{1}{2 \varepsilon}\left(\left\|p(0)-u_{0}\right\|_{3 / 2}^{2}+\left\|v_{0}\right\|_{2}^{2}\right)-\frac{\varepsilon}{2}\left(\|h\|_{3}^{2}+\|k\|_{2}^{2}\right) \\
& +\frac{1}{2}\|k\|_{2}^{2}+\frac{c_{1}}{6}\|h\|_{3}^{3}+\left(\frac{c_{1}}{6}+c\right) \iint_{\Omega}\left(h^{-}\right)^{3} d x d y
\end{aligned}
$$


for all $\varepsilon>0$. Suitable choice of $\varepsilon$ and the $L^{s}(\Omega)$-constraint for $h^{-}$due to (3.4) yields

$$
J(h, k) \geq c_{4}\left(\|h\|_{3}^{2}\left(\|h\|_{3}-1\right)+\|k\|_{2}^{2}\right)-c_{5}
$$

with positive constants $c_{4}$ and $c_{5}$.

b2) The coerciveness of the functional $J$ proved above enables us to apply the well known "trick" for minimum problem on unbounded sets (cf., e.g., [20: pp.154 - 155]). Hence,

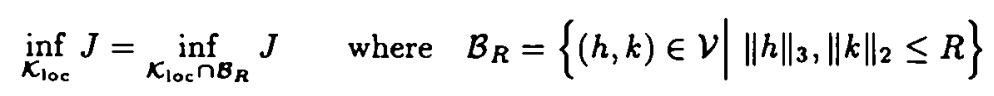

with a certain constant $R>0$. Since the set $\mathcal{K}_{\text {loc }} \cap \mathcal{B}_{R}$ is weakly compact in $\mathcal{V}$ by virtue of Lemma 3.4 and the functional $J$ is weakly lower semi-continuous on this set due to statement a) we have got again the standard situation. The existence of a solution for problem (3.3) follows immediately.

c) Let $\left\{\left(u_{n}, v_{n}\right)\right\} \subset \mathcal{K}_{\text {loc }}$ be a minimizing sequence for the variational problem (3.3). Selecting a suitable subsequence we can assume that

$$
J\left(u_{n}, v_{n}\right) \longrightarrow \alpha=\min _{\mathcal{K}_{\text {loc }}} J, \quad u_{n}-u \text { in } L^{3}(\Omega), \quad v_{n}-v \text { in } L^{2}(\Omega) .
$$

Passing to the limit $n \rightarrow \infty$ in (3.9) we obtain

$$
\alpha-J(u, v) \geq \limsup _{n \rightarrow \infty}\left(\frac{1}{2}\left\|v_{n}-v\right\|_{2}^{2}+\frac{c_{1}}{3} \iint_{\Omega^{+}} u^{+}\left(u_{n}-u\right)^{2} d x d y\right) \geq 0 .
$$

Note that $u_{n}^{+}\left(u_{n}-u\right)^{2} \geq 0$ a.e. on $\Omega$ and $u^{+}=0$ a.e. on $\Omega^{0-}$. The last terms of the right-hand side of (3.9) again converges to 0 (cf. step a2)). Since $J(u, v) \geq \alpha$, the estimate (3.11) yields $J(u, v)=\alpha$ as $v_{n} \rightarrow v$ in $L^{2}(\Omega)$, and

$$
\lim _{n \rightarrow \infty} \iint_{\Omega^{+}} u^{+}\left(u_{n}-u\right)^{2} d x d y=0
$$

i.e. convergence of $u_{n}$ to $u$ in a weighted Lebesgue space. To prove convergence in $L^{q}\left(\Omega^{+}\right)$we apply Lemma 3.6 below and get $u_{n} \rightarrow u$ in $L^{2}\left(\Omega^{+}\right)$. Interpolation and Lemma 3.4/(iii) gives the rest of the assertion

For the completeness of the proof above we have to prove the following simple lemma.

Lemma 3.6. Let $\omega \subseteq \mathbb{R}^{N}, \mu_{N}(\omega)<\infty, f_{n} \in L^{r}(\omega)$ and $f \in L^{r^{*}}(\dot{\omega})$, with $r>1$ and $\frac{1}{r}+\frac{1}{r^{*}}=1$. Suppose that $f \neq 0$ a.e. on $\omega,\left\|f_{n}\right\|_{L^{r}(\omega)} \leq R$ for all $n \in \mathbb{N}$, and

$$
\int_{\omega}\left|f f_{n}\right| d \mathbf{x} \rightarrow 0
$$

Then $f_{n} \rightarrow 0$ in $L^{1}(\omega)$ as $n \rightarrow \infty$. 
Proof. a) Defining $\omega_{m}=\left\{\mathbf{x} \in \omega|| f(\mathbf{x}) \mid \geq \frac{1}{m}\right\}$ for $m \in \mathbb{N}$ we have a monotone increasing sequence of subsets of $\omega$ :

$$
\omega_{1} \subseteq \omega_{2} \subseteq \cdots \subseteq \bigcup_{m=1}^{\infty} \omega_{m}=\omega \backslash \omega_{0} \quad \text { with } \mu_{N}\left(\omega_{0}\right)=0
$$

and hence

$$
\lim _{m \rightarrow \infty} \mu_{N}\left(\omega \backslash \omega_{m}\right)=0
$$

b) We split up $\omega=\left(\omega \backslash \omega_{m}\right) \cup \omega_{m}$ and consider the integrals over either of the two disjunct subsets:

$$
\begin{aligned}
& \cdots \int_{\omega \backslash \omega_{m}}\left|f_{n}\right| d \mathbf{x} \leq \mu_{N}\left(\omega \backslash \omega_{m}\right)^{1 / r^{*}}\left(\int_{\omega \backslash \omega_{m}}\left|f_{n}\right|^{r \cdot d \mathbf{x}}\right)^{1 / r} \leq R \mu_{N}\left(\omega \backslash \omega_{m}\right)^{1 / r^{*}} \\
& \int_{\omega_{m}}\left|f_{n}\right| d \mathbf{x} \leq m \int_{\omega_{m}}|f|\left|f_{n}\right| d \mathbf{x} \leq m \int_{\omega}\left|f f_{n}\right| d \mathbf{x} .
\end{aligned}
$$

For fixed $m$ assumption (3.12) yields

$$
\underset{n \rightarrow \infty}{\limsup } \int_{\omega}\left|f_{n}\right| d \mathbf{x} \leq R \mu_{N}\left(\omega \backslash \omega_{m}\right)^{1 / r^{*}}
$$

Finally, relation (3.13) implies the desired convergence of the sequence $\left\{f_{n}\right\}$

\section{Some properties of minimizers}

We consider a solution $(u, v) \in \mathcal{K}_{\text {loc }}$ of the minimum problem (3.3). In general it does not satisfy the corresponding variational inequality because the set $\mathcal{K}_{\text {loc }}$ is not convex. But, we can show that $(u, v)$ is a solution of a certain quasi-variational inequality (cf. [20: p. 10]) where the convex set depends on $(u, v)$. This set is defined by restrictions on the subsonic, sonic, and supersonic regions of the admissible functions.

Definition 4.1. Let $(u, v) \in \mathcal{K}_{\text {loc }}$. Using the definitions of the subsets $\Omega^{+}$and $\Omega^{0-}$ for the corresponding subsets depending on $(u, v)$ we put

$$
\mathcal{K}_{\text {loc }}[u, v]=\left\{\begin{array}{l|l}
(h, k) \in \mathcal{K}_{\text {loc }} & \begin{array}{l}
h \geq 0 \text { a.e. on } \Omega^{+} \\
h \leq 0 \text { a.e. on } \Omega^{0-}
\end{array}
\end{array}\right\} .
$$

It is immediately seen that $(u, v) \in \mathcal{K}_{\text {loc }}[u, v]$ and that $\mathcal{K}_{\text {loc }}[u, v]$ is a closed subset of $\mathcal{V}$. Furthermore, we have the following 
Theorem 4.2. Let the functional $J$ and the sets $\mathcal{K}_{\text {loc }}, \mathcal{K}_{\text {loc }}[u, v]$ be given by (2.5), (3.4) and (4.1), respectively. Then:

a) The set $\mathcal{K}_{\text {loc }}[u, v]$ is a convex subset of $\mathcal{K}_{\text {loc }}$.

b) Let the element $(u, v) \in \mathcal{K}_{\text {loc }}$ be a minimizer to problem (3.3). Then it satisfies the quasi-variational inequality (cf. (2.13))

$$
D J(u, v ; h-u, k-v) \geq 0 \quad \text { for all }(h, k) \in \mathcal{X}[u, v] .
$$

Proof. a) To prove the convexity of the set $\mathcal{K}_{\text {loc }}[u, v]$ let $\left(h_{1}, k_{1}\right),\left(h_{2}, k_{2}\right) \in \mathcal{K}[u, v]$, $\lambda \in[0,1]$ and $(h, k)=\lambda\left(h_{1}, k_{1}\right)+(1-\lambda)\left(h_{2}, k_{2}\right)$. Recalling Definition 4.1 we get

$$
\begin{array}{ll}
h \geq 0 \\
h \leq 0
\end{array} \quad \text { and } \quad \begin{array}{ll}
h^{-}=0=\lambda h_{1}^{-}+(1-\lambda) h_{2}^{-} & \text {a.e. on } \Omega^{+} \\
h^{-}=h=\lambda h_{1}^{-}+(1-\lambda) h_{2}^{-} & \text {a.e. on } \Omega^{0-} .
\end{array}
$$

Hence, $h^{-}$has the same $L^{s}(\Omega)$-bound $m$ as $h_{1}^{-}$and $h_{2}^{-}$. Moreover, since $h_{1}^{-}$and $h_{2}^{-}$ satisfy condition (3.2), the same is valid for $h^{-}$, and consequently, $(h, k) \in \mathcal{K}_{\text {loc }}[u, v]$.

b) For all elements $(h, k) \in \mathcal{K}_{\text {loc }}\{u, v]$ we have $J(u, v) \leq J(h, k)$. Together with part a) this implies inequality (4.2)

Theorem 4.3. Suppose that the element $(u, v) \in \mathcal{K}_{\text {loc }}$ is a minimizer to problem (3.3) with

$$
\tilde{\beta}:=\underset{\tilde{\Omega}}{\operatorname{essinf}} u>0
$$

where $\tilde{\Omega}$ is a subdomain of $\Omega$. Then $(u, v)$ is a solution of problem (1.8) in the distributional sense on $\tilde{\Omega}$.

Proof. For $\phi \in C_{0}^{\infty}(\tilde{\Omega})$ extended by 0 outside of $\tilde{\Omega}$ we obtain

$$
\left(h_{e}, k_{\varepsilon}\right):=(u, v)+\varepsilon\left(\phi_{x}, \phi_{y}\right) \in \mathcal{K}_{\text {loc }} \quad \text { for all } \varepsilon \in \mathbb{R},|\varepsilon| \text { sufficiently small. }
$$

To see this we observe that $\left(h_{\varepsilon}, k_{\varepsilon}\right) \in \mathcal{V}$ and

$$
\begin{aligned}
& h_{\varepsilon}=u \quad \text { and } \quad h_{e}^{-}=u^{-} \quad \text { a.e. on } \Omega \backslash \tilde{\Omega} \\
& h_{e} \geq \tilde{\beta}-|\varepsilon|\left\|\Phi_{x}\right\|_{L^{\infty}(\tilde{\Omega}}>0 \quad \text { and } \quad h_{e}^{-}=0=u^{-} \quad \text { a.e. on } \tilde{\Omega}
\end{aligned}
$$

for $|\varepsilon|<\tilde{\beta} /\left\|\phi_{x}\right\|_{L^{\infty}(\tilde{\Omega})}$. Hence, $h_{\varepsilon}^{-}=u^{-}$a.e. on $\Omega$, from which the inclusion $\left(h_{\varepsilon}, k_{\varepsilon}\right) \in$ $\mathcal{K}_{\text {loc }}$ follows. Moreover, we have $J\left(h_{\varepsilon}, k_{\varepsilon}\right) \geq J(u, v)$ for all such $\varepsilon$, and consequently, $D J\left(u, v ; \phi_{x}, \phi_{y}\right)=0$ for all $\phi \in C_{0}^{\infty}(\tilde{\Omega})$. Using the definitions of $\mathcal{V}$ in $(2.1)$, of $D J$ in (2.13), and of $\left(u_{0}, v_{0}\right)$ in (2.3) we get the assertion

Remark 4.4. The result in the last theorem is one advantage of the concept of local entropy conditions used above. It says that on each subdomain of $\Omega$ in which a minimizer to problem (3.3) is uniformly subsonic (i.e. uniformly positive) it is a weak solution to our original problem (1.8). In the case that it is uniformly subsonic in the whole of $\Omega$, in addition, a uniqueness result can be derived. 
Theorem 4.5. Let the element $(u, v) \in \mathcal{K}_{\text {loc }}$ be a minimizer to problem (3.3) with

$$
\beta:=\underset{\Omega}{\operatorname{essinf}} u>0 \text {. }
$$

Then:

a) $(u, v)$ is a generalized solution of the boundary value problem (1.8),(1.9) (cf. Definition 2.1). with

b) Moreover, there is no other solution $(\tilde{u}, \tilde{v}) \in \mathcal{K}_{\text {loc }}$ of the minimum problem (3.3)

$$
\underset{\Omega}{\operatorname{essinf}} \tilde{u}>-2 \beta \frac{c_{1}}{c_{1}+\left(c_{2}-c_{1}\right)^{+}} \text {. }
$$

Proof. a) Analogously to the proof of Theorem 4.3 we get $(u, v)+\varepsilon(h, k) \in \mathcal{K}_{\text {loc }}$ for all $\varepsilon \in \mathbb{R},|\varepsilon|$ sufficiently small, and all $(h, k) \in \mathcal{V}$. Hence, $D J(u, v ; h, k)=0$, or equivalently (2.4) follows.

b) Let $(\tilde{u}, \tilde{v}) \in \mathcal{K}_{\text {loc }}$ be another minimizer to problem (3.3) satisfying condition (4.4). The estimate $(2.8)$ yields

$$
\begin{aligned}
0 & =J(\tilde{u}, \tilde{v})-J(u, v) \\
& \geq D J(u, v ; \tilde{u}-u, \tilde{v}-v)+\frac{1}{2}\|\tilde{v}-v\|_{2}^{2} \\
& +\frac{1}{6} \iint_{\Omega}\left(c_{1}(\tilde{u}+2 u)+\left(c_{2}-c_{1}\right)^{+}\left(\tilde{u}^{-}+2 u^{-}\right)\right)(\tilde{u}-u)^{2} d x d y .
\end{aligned}
$$

Using (2.4) and (4.3),(4.4) we obtain that the right-hand side of this inequality is positive if $(\tilde{u}, \tilde{v}) \neq(u, v)$

Remark 4.6. i) The last proof shows that the uniqueness result (Theorem $4.5 . / b$ )) is also valid if the set $\mathcal{K}_{\text {loc }}$ is substituted by $\mathcal{K}$. Note that in this case the variational inequality (2.13) holds.

ii) We want to point out that the same methods presented in this paper work in higher dimensions if we start with the system

$$
\begin{aligned}
& \frac{\partial}{\partial x_{1}} p\left(u_{1}\right)+\sum_{i=2}^{N} \frac{\partial}{\partial x_{i}} u_{i}=\operatorname{div}\left(p\left(u_{1}\right), u_{2}, \ldots, u_{N}\right)=0 \\
& \operatorname{rot} \mathbf{u}=0, \quad \text { i.e. } \frac{\partial}{\partial x_{i}} u_{j}=\frac{\partial}{\partial x_{j}} u_{i} \text { for all } i, j=1, \ldots, N
\end{aligned}
$$

for the unknown $\mathbf{u}=\left(u_{1}, \ldots, u_{N}\right) \in \mathbb{R}^{N}$. This system is studied in a bounded domain $\omega \subseteq \mathbb{R}^{N}$ with piecewise smooth boundary where appropriate boundary conditions are laid on $\mathbf{u}$. Here, $p$ is again a given smooth function on $\mathbb{R}$ satisfying the assumptions (1.10) and (1.11). For example, such boundary value problems with $p\left(u_{1}\right)=\frac{1}{2} u_{1}^{2}$ occur if the transonic expansion procedure from Section 1 is applied to three-dimensional wings (cf. [1: Subsection 3.1.1]). We omit the details.

Acknowledgement. This research was supported in part by the Deutsche Forschungsgemeinschaft (DFG) via "Forschergruppe: Nichtlineare Funktionalanalysis und Mathematische Methoden der Kontinuumsmechanik". The paper was completed during a stay at the Courant Institute, New York University. In particular, we thank Prof. Cathleen Morawetz for helpful discussions. 


\section{References}

[1] Cole, J. D. and L. P. Cook: Transonic Aerodynamics. Amsterdam: North-Holland Publ. Comp. 1986.

[2] Feistauer, M. and J. Nečas: On the solvability of transonic potential flow problems. Z. Anal. Anw. 4 (1985), 305 - 329.

[3] Feistauer, M. and J. Necias: On the solution of transonic flows with weak shocks. Comment. Math. Univ. Carolin. 27 (1986), 791 - 804.

[4] Feistauer, M. and J. Nečas: Viscosity method in a transonic flow. Comm. Part. Diff. Equ. 13 (1988), 775 - 812.

[5] Feistauer, M. and J. Nećas: Remarks on the solvability of transonic flow problems. Manuscr. Math. 61 (1988), $417-428$.

[6] Gittel, H.-P.: Studies on transonic flow problems by nonlinear variational inequalities. Z. Anal. Anw. 6 (1987), 449 - 458.

[7] Gittel, H.-P.: Local entropy conditions in transonic potential flow problems. Math. Nachr. 154 (1991), 117 - 127.

[8] Landau, L. D. and E. M. Lifschitz: Lehrbuch der Theoretischen Physik. Vol. VI: Hydrodynamik. Berlin: Akademie-Verlag 1966.

[9] Morawetz, C. S.: On a weak solution for a transonic flow problem. Comm. Pure Appl. Math. 38 (1985), $797-818$.

[10] Morawetz, C. S.: On steady transonic flow by compensated compactness. Comm. Pure Appl. Math. (to appear).

[11] Murat, F.: L'injection du cône positif de $H^{-1}$ dans $W^{-1, q}$ est compacte pour tout $q<2$. J. Math. Pures Appl. 60 (1981), 309 - 322.

[12] Pogu, M. and J. E. Souza de Cursi: Contribution a l'etude pe problemes aux limites non monotones stationnaires. Publications of the Service de Mathématiques. École Nationale Supérieure de Méchanique Nantes 1989.

[13] Pogu, M. and G. Tourmine: Une methode functionnelle de resolution approchee d'un probleme transonique. C. R. Acad. Sci. Paris 312, Série II (1991), 431 - 434.

[14] Pogu, M. and G. Tourmine: An approached functional solution of the Karman-Guderley equation. Publications of the Service de Mathématiques. École Nationale Supérieure de Méchanique Nantes 1992.

[15] Pogu, M. and G. Tourmine: A functional approach to the solution of the Karman-Guderley equation. Bull. Polish Acad. Sci. Tech. Sci. 40 (1992), 335 - 344.

[16] Smoller, J.: Shock Waves and Reaction-Diffusion Equations. New York: Springer-Verlag 1983.

[17] Struwe, M.: Variational Methods: Applications to Nonlinear Partial Differential Equations and Hamiltonian Systems. Berlin: Springer-Verlag 1990.

[18] Temam, R.: Navier-Stokes Equations: Theory and Numerical Analysis. Amsterdam: North-Holland Publ. Comp. 1977.

[19] Warnecke, G.: Admissibility of solutions to the Riemann problem for systems of mixed type - transonic small disturbance theory. In: Nonlinear Evolution Equations that Change Type (eds.: B. L. Keyfitz and M. Shearer). The IMA Volumes in Mathematics and Its Applications: Vol. 27. New York: Springer-Verlag 1990, pp. 258-284.

[20] Zeidler, E.: Nonlinear Functional Analysis and Its Applications. Vol. III: Variational Methods and Optimization. New York: Springer-Verlag 1985. 